\title{
ANÁLISE DE ESCALA DIAGRAMÁTICA PARA AVALIAÇÃO DA SEVERIDADE DA ANTRACNOSE DAS VAGENS DE FEIJÃO-FAVA
}

\author{
Frederico Monteiro Feijón ${ }^{*}$, Wellington Costa da Silva², Iraildes Pereira Assunção', Ricardo Brainer \\ Martins $^{3}$, Sami Jorge Michereff ${ }^{4}$, Gaus Silvestre de Andrade Lima ${ }^{1}$
}

\begin{abstract}
${ }^{1}$ Centro de Ciências Agrárias, Universidade Federal de Alagoas, BR 104 Norte, Km 85, Rio Largo, Alagoas. ${ }^{2}$ Instituto Federal de Pernambuco, Campus Vitoria de Santo Antão, 55602-970 Vitoria de Santo Antão, Pernambuco. ${ }^{3}$ Campus Arapiraca, Universidade Federal de Alagoas, 57309-005 Arapiraca, Alagoas. Departamento de Agronomia, Universidade Federal Rural de Pernambuco, 52171900 Recife, Pernambuco.

*Autor para correspondência: Frederico Monteiro Feijó, frederico.agro2004@gmail.com
\end{abstract}

RESUMO: A antracnose das vagens, causada pelo fungo Colletotrichum truncatum, é uma importante doença do feijão-fava no Nordeste brasileiro. Devido à inexistência de métodos padronizados para quantificação desta doença no campo, uma escala diagramática desenvolvida para avaliação da severidade da antracnose em vagens do feijão-comum, com níveis de severidade variando de 1,5 a 34\%, foi testada para acurácia, precisão, repetibilidade e reprodutibilidade das estimativas de severidade da antracnose em vagens de feijão-fava. No teste de validação da escala diagramática, 50 vagens de feijão-fava com diferentes níveis de severidade da doença, mensurados previamente com o programa ASSESS, foram avaliadas por 10 avaliadores, sem e com a utilização da escala diagramática. Foram realizadas duas avaliações com a utilização da escala, com intervalo de uma semana. A acurácia e a precisão de cada avaliador foram determinadas por regressão linear simples, entre a severidade real e a estimada. A utilização da escala diagramática não propiciou redução consistente dos erros absolutos dos avaliadores, que se concentraram na faixa entre 10 e $30 \%$. 0 uso da escala diagramática na primeira e na segunda avaliação não resultou em reduções significativas dos erros sistemáticos e nem em melhoria na acurácia e precisão das estimativas quando comparado à avaliação sem o uso da escala. Além disso, os avaliadores não evidenciaram boa repetibilidade e reprodutibilidade nas estimativas da severidade da antracnose em vagens de feijão-fava com o uso da escala diagramática. Portanto, torna-se essencial o desenvolvimento e a validação de uma escala diagramática específica para quantificação da antracnose em vagens de feijão-fava.

PALAVRAS-CHAVE: Phaseolus lunatus, Colletotrichum truncatum, patometria.

\section{ANALYSIS OF DIAGRAMMATIC KEY FOR ASSESSMENT OF ANTHRACNOSE SEVERITY IN LIMA BEAN PODS}

ABSTRACT: Anthracnose of pods caused by the fungus Colletotrichum truncatum is an important disease of lima bean in Brazilian northeastern. Due to the inexistence of standard methods to quantify this disease in field conditions, a diagrammatic key developed to assessment of severity anthracnose in common bean pods, with severity levels ranging from 1.5 to $34 \%$, it was tested for accuracy, precision, repeatability and reproducibility of anthracnose severity estimates in lima bean pods. The diagrammatic key was validated by 10 raters without and with using the key, using 50 pods with different levels of severity previously measured by the software ASSESS ${ }^{\circledR}$. Two evaluations were performed with the use of the key, with an interval of one week. The accuracy and precision of each rater were determined by simple linear regression between actual and estimated severity. The use of diagrammatic did not provide consistent reduction of the absolute errors of the raters, who concentrated in the range between 10 and $30 \%$. The use of diagrammatic key in the first and second evaluation did not result in significant reductions in systematic errors and not on improving the accuracy and precision of estimates when compared to the evaluation without the use of the key. In addition, the evaluators did not show good repeatability and reproducibility in the estimates of the severity of anthracnose in lima bean pods using the diagrammatic key. Therefore, it is essential to the development and validation of a specific diagrammatic key to quantify the anthracnose in lima bean pods.

KEYWORDS: Phaseolus lunatus, Colletotrichum truncatum, pathometry. 


\section{INTRODUÇÃO}

O feijão-fava (Phaseolus lunatus L.) apresenta relevância no contexto da agricultura familiar no Nordeste brasileiro, constituindo importante alternativa de renda e fonte de alimento para a população (Guimarães et al., 2007; Silva et al., 2010). Essa região é responsável por mais de $98 \%$ da produção brasileira de feijão-fava, que alcançou em 2015 uma área plantada de 21.047 ha e produção de 3.977 toneladas (IBGE, 2016).

A ocorrência de doenças tem sido apontada como um dos fatores de redução da produtividade $e$ da qualidade do feijão-fava produzido no Nordeste brasileiro, com destaque para a antracnose, causada pelo fungo Colletotrichum truncatum (Schw.) Andrus \& Moore (Cavalcante et al., 2012). A doença atinge folhas, ramos e vagens, podendo causar redução da produtividade e queda do valor comercial dos grãos. Os principais sintomas são lesões deprimidas nas vagens, com coloração marrom-escura e bordas vermelho-arroxeadas. Nas folhas são observadas lesões avermelhadas, inicialmente nas nervuras da parte inferior, seguido de necrose do limbo, tanto nas faces adaxial quanto na abaxial. Hastes, pecíolos jovens e hipocótilo também podem apresentar lesões avermelhadas e deprimidas (Paula Júnior et al., 1995).

Apesar da importância da antracnose em feijãofava, até o momento inexistem estudos epidemiológicos dessa doença. Esses estudos requerem métodos padronizados para quantificação da doença no campo, que devem ser simples e de rápida utilização, bem como acurados, precisos e reprodutíveis. A acurácia representa 0 grau de proximidade entre a estimativa média e a quantidade real de doença, a precisão referese à confiabilidade e/ou repetibilidade nas avaliações da doença e a reprodutibilidade indica a ausência de variação em estimativas quando a mesma amostra de doença é avaliada por outro avaliador (Campbell e Madden, 1990).

Considerando que o principal sintoma da antracnose do feijão-fava é na vagem, sua intensidade deve ser determinada pela severidade, ou seja, pela porcentagem ou proporção de área da vagem lesionada. $\mathrm{Na}$ avaliação da severidade da antracnose em vagens de feijão-fava têm sido utilizadas apenas escalas descritivas (Carvalho, 2009; Cavalcante et al., 2012; Carmo et al., 2015), que apresentam a inconveniência de serem altamente subjetivas e não permitirem um ajuste da acuidade visual na avaliação dos níveis de severidade (Campbell e Madden, 1990). Diante disso, a avaliação da severidade dessa doença com o auxílio de uma escala diagramática pode constituir uma opção para estudos de campo.

Escalas diagramáticas são representações ilustradas de uma série de partes de plantas ou de plantas inteiras com sintomas em diferentes níveis de severidade (Campbell e Madden, 1990). Atualmente, essas escalas se constituem na principal ferramenta de avaliação da severidade para muitas doenças (Bock et al., 2010; Duarte et al., 2014; Lopes et al., 2014; Michereff e Correia, 2016). A simples utilização de uma escala diagramática não garante melhoria na qualidade das estimativas da severidade de uma doença, sendo necessário que essa escala seja submetida a um processo de validação. Para validação de uma escala diagramática, os valores de severidade real em plantas ou partes de plantas devem ser obtidos com precisão, diversos avaliadores devem quantificar a severidade da doença sem e com o auxílio da escala diagramática e, finalmente, devem ser comparados os níveis de acurácia, precisão e reprodutibilidade das estimativas propiciados sem e com a utilização da escala (Madden et al., 2007; Bock et al., 2010).

Não existe escala diagramática para quantificação da severidade da antracnose em vagens de feijão-fava, mas existe uma escala para avaliação da antracnose em vagens do feijão-comum (Phaseolus vulgaris L.) (Castaño-Zapata, 1989). № entanto, essa escala não foi submetida ao processo de validação, motivo pelo qual é desconhecido se a utilização da mesma propiciará melhorias na qualidade das estimativas de severidade. Diante disso, este trabalho teve como objetivo analisar os níveis de acurácia, precisão e reprodutibilidade das estimativas de severidade da antracnose em vagens de feijão-fava com a utilização da escala desenvolvida para avaliação da antracnose em vagens do feijão-comum.

\section{MATERIAL E MÉTODOS \\ Obtenção da severidade real}

Uma amostra de 200 vagens de feijão-fava (cv. 'Rajada') com diferentes níveis de severidade da antracnose foi coletada em campo experimental do Centro de Ciências Agrárias da Universidade Federal de Alagoas (CECA/UFAL), localizado no município de Rio Largo, Alagoas. Imagens digitais foram capturadas 
a partir de uma visão lateral de cada uma das vagens usando uma câmera fotográfica digital (Canon PowerShot S70, 7.1 megapixels) montada a $20 \mathrm{~cm}$ de altura de cada vagem. Cada imagem foi analisada com o programa ASSESS versão 2.0 (Lamari, 2008). A severidade real da antracnose nas vagens foi obtida automaticamente, baseada no modelo de cor Matiz-Saturação-Intensidade (HSI), que determina a porcentagem entre área total e área doente da vagem em pixels. Um pesquisador experiente na avaliação da doença selecionou uma sub-amostra de 50 vagens com sintomas representando o intervalo entre os níveis mínimo e máximo de severidade na amostra. Este subconjunto foi utilizado para todas as avaliações neste trabalho.

\section{Teste de validação da escala diagramática}

A escala diagramática para avaliação da severidade da antracnose em vagens de feijão-comum (Figura 1), desenvolvida por Castaño-Zapata (1989), com cinco níveis de severidade variando de 1,5 a 34\%, foi submetida ao processo de validação. No teste de validação foi utilizado o subconjunto, previamente selecionado, de 50 imagens digitalizadas de feijãofava com sintomas de antracnose em diferentes níveis de severidade. Cada imagem foi inserida em um único slide de arquivo de apresentação do Microsoft PowerPoint. Dez avaliadores (A a J) sem experiência na quantificação de doenças de plantas foram selecionados e instruídos sobre os sintomas de antracnose nas vagens de feijão-fava e como realizar a avaliação da severidade da doença. Durante cada sessão, as imagens digitais das vagens doentes foram projetadas em uma sequência aleatória durante 30 segundos por imagem. Todos os avaliadores indicaram simultaneamente a porcentagem de severidade em três sessões de avaliação. Na primeira sessão, a severidade da antracnose em cada vagem foi estimada diretamente, sem qualquer auxílio (Sem escala). Uma semana depois, os avaliadores efetuaram as estimativas com 0 auxílio da escala diagramática (Com Escala $1^{\text {a }}$ Avaliação), após receberem instruções sobre como usarem a escala considerando a porcentagem mais próxima, ou seja, usarem os valores da escala como pontos de referência para auxiliar nas estimativas. Após um novo intervalo de uma semana, novas estimativas foram realizadas com o auxílio da escala diagramática (Com Escala $2^{\mathrm{a}}$ Avaliação).
Figura 1. Escala diagramática para avaliação da severidade da antracnose em feijão-comum, indicando os níveis de 1,5, 6, 11, 21 e $34 \%$ de severidade (Castaño-Zapata, 1989).
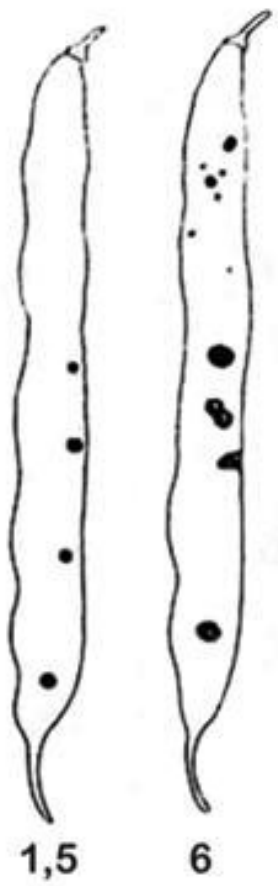

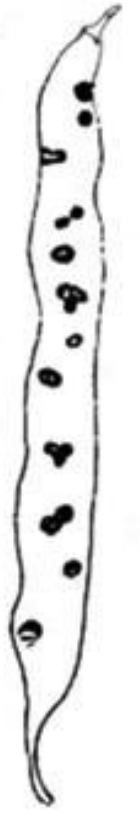

11
21

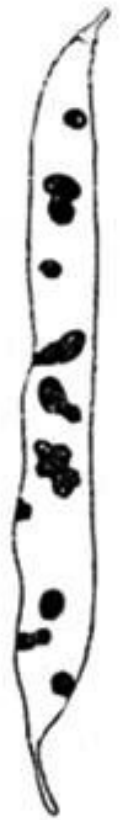

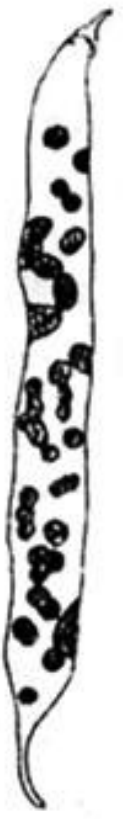

34
A acurácia e a precisão de cada avaliador foram estimadas por regressão linear simples, sendo a variável independente a severidade real, obtida com o programa ASSESS, e a variável dependente a severidade estimada pelo avaliador. A acurácia das estimativas de cada avaliador e do conjunto de avaliadores foi determinada pelo teste $t$ aplicado ao intercepto da regressão linear (a), para verificar a hipótese Ho: $a=0$, e ao coeficiente angular da reta $(b)$, para testar a hipótese Ho: $b=1$, ao nível $5 \%$ de probabilidade $(\mathrm{P}=0,05)$. Valores de intercepto significativamente diferentes de 0 (zero) indicam superestimativa $(>0)$ ou subestimativa $(<0)$ da severidade real a níveis baixos de intensidade da doença, enquanto valores de coeficiente angular da reta que desviam significativamente de 1 (um) indicam superestimativa $(>1)$ ou subestimativa $(<1)$ sistemática da severidade real em todos os níveis de intensidade da doença. A precisão das estimativas foi determinada pelo coeficiente de determinação da regressão $\left(R^{2}\right)$, pela variância dos erros absolutos (severidade estimada menos severidade real) e pela repetibilidade das estimativas, determinada pela regressão da segunda avaliação em relação à primeira de uma mesma unidade amostral. A reprodutibilidade das estimativas foi determinada pelos valores de $R^{2}$ obtidos 
de regressões lineares entre as severidades estimadas da mesma unidade amostral por diferentes avaliadores combinados em pares (Campbell e Madden, 1990; Nutter Jr. e Schultz, 1995). As análises de regressão foram efetuadas com o auxílio do programa Microsoft Office Excel 2007 (Microsoft Corporation, Redmont, WA, USA).

\section{RESULTADOS E DISCUSSÃO}

A severidade real da antracnose na amostra de 200 vagens de feijão-fava coletadas no campo variou de 0,37 a $96,14 \%$, sendo que $27,6 \%$ das vagens tinham lesões que totalizavam mais de $40 \%$ de severidade (Figura 2A). Na sub-amostra, utilizada em todas as etapas de validação, a distribuição entre os diferentes níveis foi mais homogênea, sendo este um dos critérios para seleção das mesmas. Assim, obedecendo ao intervalo de 0,37 a $96,14 \%$ de severidade, a subamostra teve a seguinte distribuição: 9 vagens até $20 \%$ de severidade da doença, 12 vagens com severidade entre 21 e 40\%, 14 vagens com severidade entre 41 e $60 \%, 9$ vagens com severidade entre 61 e $80 \%$ e 6 vagens com severidade superior a $80 \%$ (Figura 2B). A escala submetida à validação considera $34 \%$ o nível máximo de severidade da antracnose nas vagens, indicando inadequação quando comparada aos níveis observados nas vagens de feijão-fava, tanto da amostra, onde $27,6 \%$ destas apresentaram severidade superior a $40 \%$, quanto da sub-amostra, que totalizou $58 \%$ de vagens com severidade superior a $40 \%$. Esse aspecto é relevante, pois na elaboração da escala diagramática deve ser considerado o limite máximo da severidade da doença observada no campo (Kranz, 1988; Campbell e Madden, 1990; Nutter Jr. e Schultz, 1995).

Figura 2. Distribuição de frequência de vagens de feijão-vagem com diferentes intervalos de severidade da antracnose considerando: A - amostra total com 200 vagens, e B - sub-amostra utilizada para validação com 50 vagens.

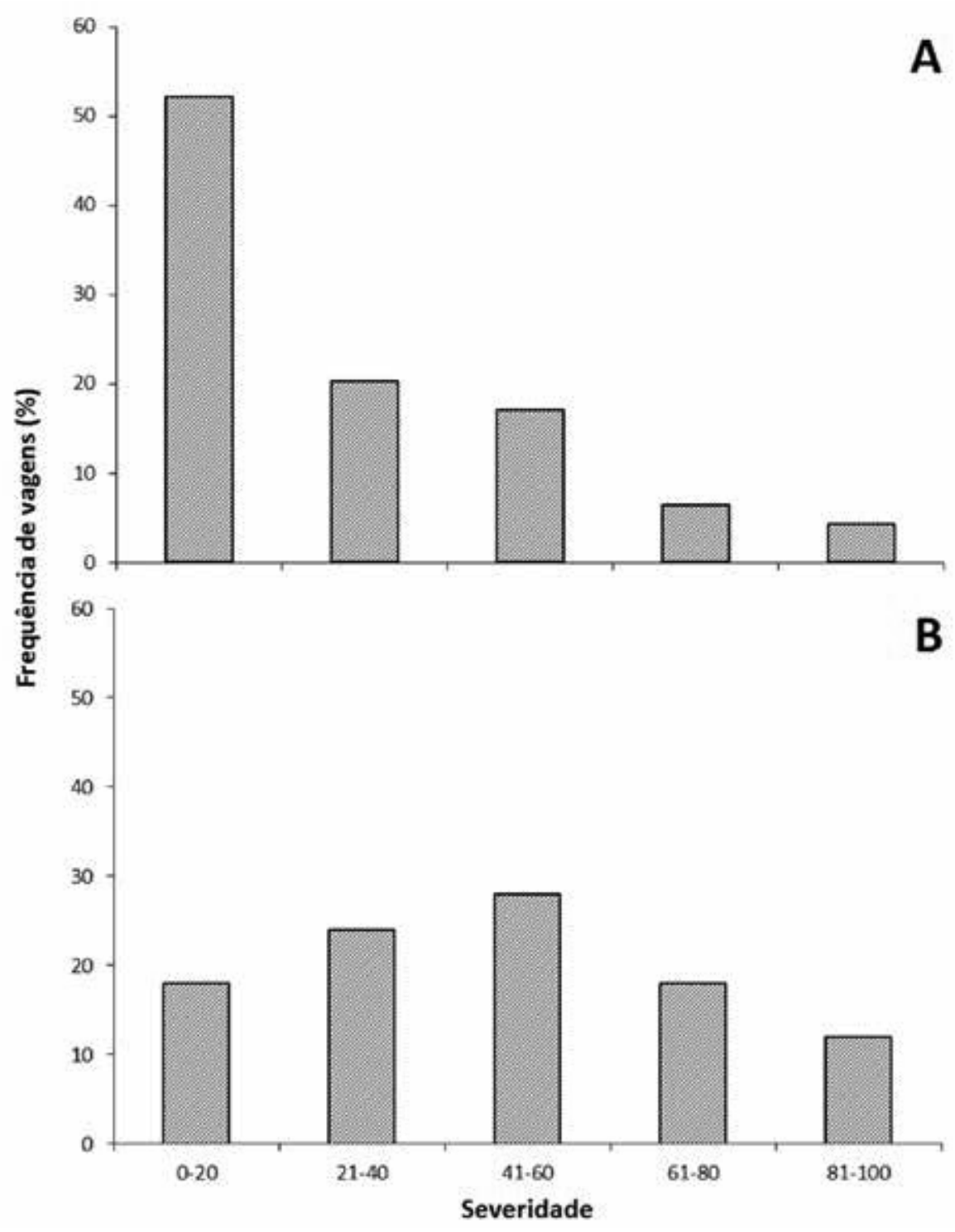


Os valores de severidade real da antracnose nas 50 vagens de feijão-fava coletadas no campo variaram de 0,37 a $96,14 \%$, sendo que 21 vagens (42\%) apresentaram até $40 \%$ de severidade da doença, enquanto 15 vagens (30\%) apresentaram severidade superior a $60 \%$ (Figura 2).

Quando a severidade da antracnose nas vagens de feijão-fava foi estimada sem 0 auxílio da escala, sete avaliadores foram pouco acurados apresentando valores do intercepto significativamente diferentes de zero para as retas de regressão entre a severidade real e a estimada, com valor médio de 3,21 (Tabela 1). Cinco desses avaliadores ( $B, C, G, H$ e J) superestimaram consistentemente a severidade, indicando a presença de desvios positivos constantes, enquanto dois (A e $E)$ subestimaram a severidade. $O$ coeficiente angular da reta, na média dos avaliadores, foi de 1,01 e não diferiu significativamente de 1 (Tabela 1). Somente um avaliador $(\mathrm{J})$ apresentou valor do coeficiente angular significativamente diferente de 1 , indicando a presença de desvio sistemático em todos os níveis de intensidade da doença (Tabela 1). A precisão das estimativas sem 0 auxílio da escala foi boa, pois os coeficientes de determinação variaram entre 0,77 e 0,88 , com média de 0,84 . A distribuição dos resíduos das avaliações realizadas sem 0 auxílio da escala diagramática apresentou estimativas com erros absolutos variando entre $-29,86$ e 31,62 (Figura 3A).

Tabela 1. Acurácia e precisão das estimativas da severidade da antracnose em vagens de feijão-fava, representadas pelo intercepto (a), coeficiente angular da reta $(b)$ e coeficiente de determinação $\left(R^{2}\right)$ de equações de regressão linear simples relacionando estimativas visuais da severidade da doença efetuadas por 10 avaliadores, sem e com 0 auxílio de escala diagramática, à severidade real determinada eletronicamente com auxílio do programa ASSESS .

\begin{tabular}{|c|c|c|c|c|c|c|c|c|c|}
\hline \multirow{3}{*}{ Avaliador } & \multicolumn{3}{|c|}{ Sem Escala } & \multicolumn{6}{|c|}{ Com Escala } \\
\hline & & & & \multicolumn{3}{|c|}{ 1a Avaliação } & \multicolumn{3}{|c|}{ 2a Avaliação } \\
\hline & $a$ & $b$ & $R^{2}$ & $a$ & $b$ & $R^{2}$ & $a$ & $b$ & $R^{2}$ \\
\hline$A$ & $-5,64^{*}$ & 1,10 & 0,80 & $6,51^{*}$ & 0,94 & 0,80 & $-0,91$ & 1,04 & 0,84 \\
\hline B & $12,96^{*}$ & 0,99 & 0,88 & $9,39 *$ & 0,93 & 0,81 & $-4,65$ & 1,00 & 0,79 \\
\hline C & $6,71^{*}$ & 1,05 & 0,86 & $14,83^{*}$ & $0,82^{*}$ & 0,56 & 2,65 & 1,04 & 0,90 \\
\hline D & 0,08 & 1,09 & 0,77 & 1,80 & 0,92 & 0,81 & $-0,10$ & 1,04 & 0,83 \\
\hline$E$ & $-6,18^{*}$ & 1,03 & 0,85 & $-1,31$ & $0,81^{*}$ & 0,79 & $-6,64^{*}$ & $0,86^{*}$ & 0,80 \\
\hline$F$ & $-2,82$ & 1,09 & 0,87 & 0,25 & 0,92 & 0,77 & 1,21 & 0,94 & 0,77 \\
\hline G & $10,65^{*}$ & 0,97 & 0,85 & $22,91^{*}$ & $0,67^{*}$ & 0,53 & $7,82^{*}$ & 0,98 & 0,83 \\
\hline $\mathrm{H}$ & $8,70^{*}$ & 0,94 & 0,82 & $7,85^{*}$ & $0,79^{*}$ & 0,69 & 0,92 & 1,00 & 0,88 \\
\hline I & 1,10 & 1,01 & 0,87 & $15,52^{*}$ & $0,76^{*}$ & 0,67 & $10,12^{*}$ & $0,82^{*}$ & 0,88 \\
\hline$J$ & $6,59^{*}$ & $0,89^{*}$ & 0,88 & 0,18 & $0,88^{*}$ & 0,83 & 0,27 & 0,99 & 0,83 \\
\hline Média & 3,21 & 1,01 & 0,84 & 7,75 & 0,84 & 0,73 & 1,07 & 0,97 & 0,83 \\
\hline
\end{tabular}

${ }^{\star}$ Asterisco representa situações onde a hipótese de nulidade ( $a=0$ ou $b=1$ ) foi rejeitada pelo teste $t(P=0,05)$.

$\mathrm{Na}$ primeira avaliação com a escala diagramática, os avaliadores se mantiveram pouco acurados, pois seis (A, B, C, G, H e I) apresentaram valores do intercepto significativamente diferentes de zero, todos com tendência à superestimativa dos níveis de severidade da doença. 0 valor médio do intercepto $(7,75)$ foi bem superior ao constatado sem a utilização da escala, indicando maior tendência à superestimativa (Tabela 1). 0 coeficiente angular da reta na primeira avaliação com a escala, na média dos avaliadores, foi de 0,84 e diferiu significativamente de 1 (Tabela 1). Seis avaliadores (C, E, G, H, I e J) apresentaram valores do coeficiente angular significativamente diferentes de 1 , indicando a presença de desvios sistemáticos em todos os níveis de severidade da doença (Tabela 1). A precisão das estimativas na primeira avaliação com a escala diagramática foi baixa, pois os coeficientes de determinação variaram entre 0,53 e 0,83, com média de 0,73. A distribuição dos resíduos das avaliações realizadas sem 0 auxílio da escala diagramática apresentou estimativas com erros absolutos variando entre -38,64 e 29,62 (Figura 3B).

$\mathrm{Na}$ segunda avaliação com a escala diagramática, os avaliadores melhoraram a acurácia das estimativas, pois o valor médio do intercepto $(1,07)$ não diferiu significativamente de zero e foi bem inferior ao constatado sem a utilização da escala e na primeira avaliação com a escala. Três avaliadores apresentaram valores do intercepto 
significativamente diferentes de zero, sendo que dois ( $G$ e I) superestimaram consistentemente os níveis de severidade da doença e um $(E)$ subestimou a severidade da doença. $O$ coeficiente angular da reta na segunda avaliação com a escala, na média dos avaliadores, foi de 0,97 e não diferiu significativamente de 1. Dois avaliadores ( $E$ e I) apresentaram valores do coeficiente angular significativamente diferentes de 1 (Tabela 1). Na segunda avaliação com a escala os coeficientes de determinação variaram entre $0,77 \mathrm{e}$ 0,90 , com média de 0,83. A distribuição dos resíduos das avaliações realizadas sem o auxílio da escala diagramática apresentou estimativas com erros absolutos variando entre $-44,95$ e 31,99 (Figura 3C).

Figura 3. Distribuição dos resíduos (severidade estimada - severidade real) das estimativas da antracnose em vagens de feijão-fava realizadas sem o auxílio da escala diagramática (A) e com auxílio da escala diagramática na primeira (B) e segunda (C) avaliação.
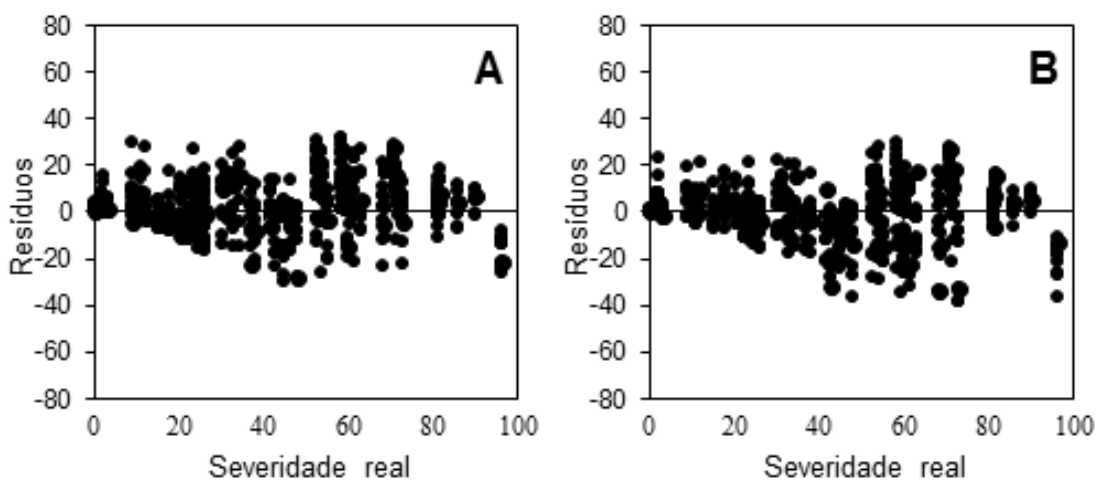

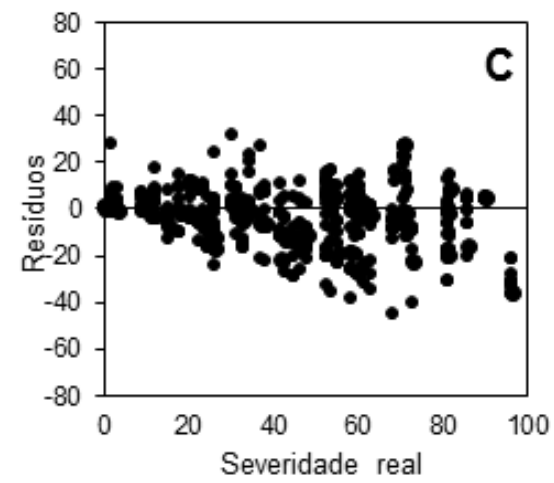

A utilização da escala não propiciou redução consistente dos erros absolutos dos avaliadores, que se concentraram na faixa entre 10 e $30 \%$. A presença de algum nivel de erro absoluto nas mensurações pode ser compensada pela rapidez e padronização propiciadas pelo uso de escalas diagramáticas (Nutter Jr. e Schultz, 1995), o que não foi constatado nesse estudo.

A tendência à superestimativa dos níveis de severidade das doenças sem 0 uso de escalas diagramáticas é comum em vários patossistemas (Kranz, 1988; Bock et al., 2010). Além disso, a tendência dos avaliadores em superestimarem os níveis de severidade da antracnose em vagens de feijão-fava quando utilizaram a escala diagramática assemelha-se ao constatado em vários estudos envolvendo validação de escalas diagramáticas (Leite e Amorim, 2002; Sposito et al., 2004; Barbosa et al., 2006; Celoto e Papa, 2010; Lima et al., 2011; Buffara et al., 2014).

O uso da escala diagramática na primeira e na segunda avaliação não resultou em reduções significativas dos erros sistemáticos e nem na melhoria na acurácia das estimativas quando comparado à avaliação sem o uso da escala. Além disso, o uso da escala não propiciou melhorias significativas na precisão das estimativas, pois sem 0 uso da escala a precisão foi similar à observada com a utilização da escala na segunda avaliação e muito superior à observada com a utilização da escala na primeira avaliação.

Os avaliadores não evidenciaram boa repetibilidade nas estimativas da severidade da antracnose em vagens de feijão-fava com 0 uso da escala diagramática, pois a quantidade média de variação na primeira avaliação explicada pela segunda avaliação foi de $77 \%$ (Tabela 2). Os valores médios do intercepto e do coeficiente angular da reta diferiram significativamente de zero e 1, respectivamente. Entre as duas avaliações, somente para dois avaliadores ( $\mathrm{F} \mathrm{e} \mathrm{J}$ ) o valor do intercepto não foi significativamente diferente de zero e para três avaliadores ( $\mathrm{E}, \mathrm{Fe} \mathrm{I})$ os valores do coeficiente angular da reta não foram significativamente diferentes de 1 , indicando a baixa precisão das estimativas para a maioria dos avaliadores. 
Tabela 2. Repetibilidade das estimativas da severidade da antracnose em vagens de feijão-fava, representada pelo intercepto (a), coeficiente angular da reta (b) e coeficiente de determinação (R2) de equações de regressão linear simples relacionando estimativas da severidade da doença efetuadas por um mesmo avaliador, com 0 auxílio da escala diagramática, em duas avaliações separadas por uma semana.

\begin{tabular}{cccc}
\hline Avaliador & $a$ & $b$ & $\mathrm{R} 2$ \\
\hline A & $9,52^{*}$ & $0,86^{*}$ & 0,86 \\
B & $18,33^{*}$ & $0,82^{*}$ & 0,80 \\
C & $13,51^{*}$ & $0,78^{*}$ & 0,60 \\
D & $7,18^{*}$ & $0,78^{*}$ & 0,75 \\
E & $6,35^{*}$ & 0,90 & 0,90 \\
F & 2,08 & 0,90 & 0,86 \\
G & $18,22^{*}$ & $0,67^{*}$ & 0,61 \\
H & $8,73^{*}$ & $0,76^{*}$ & 0,72 \\
I & $6,37^{*}$ & 0,92 & 0,75 \\
J & 2,24 & $0,83^{*}$ & 0,89 \\
\hline Média & 9,25 & 0,82 & 0,77 \\
\hline
\end{tabular}

${ }^{*}$ Asterisco indica que a hipótese de nulidade $(a=0$ ou $b=1)$ foi rejeitada pelo teste $t(P=0,05)$.

A reprodutibilidade das avaliações da severidade sem a utilização da escala diagramática foi superior à verificada com a utilização da escala. Sem a utilização da escala as regressões lineares entre as severidades estimadas pelos 10 avaliadores produziram coeficientes de determinação $\geq 0,90 \mathrm{em}$ $73,23 \%$ dos casos (Tabela 3 ). Por outro lado, com a utilização da escala os valores do coeficiente de determinação foram $\geq 0,90$ em $36,0 \%$ e $49,0 \%$ dos casos na primeira e segunda avaliação, respectivamente (Tabela 3). Portanto, o uso da escala diagramática não aumentou a reprodutibilidade das estimativas obtidas por diferentes avaliadores e, do ponto de vista prático, diferentes experimentos, conduzidos por avaliadores distintos, não serão comparáveis com a utilização da escala diagramática.

Tabela 3. Reprodutibilidade das estimativas da severidade da antracnose em vagens de feijão-fava, representada pelo coeficiente de determinação (R2) de equações de regressão linear simples relacionando as estimativas de severidade entre avaliadores sem o auxílio da escala diagramática e com auxílio da escala diagramática.

\begin{tabular}{cccc}
\hline \multirow{2}{*}{$\begin{array}{c}\text { Intervalos do coeficiente } \\
\text { de determinação (R2) }\end{array}$} & Sem Escala & \multicolumn{2}{c}{ Frequência $(\%)^{*}$} \\
\cline { 2 - 4 } & 26,7 & 1a Avaliação & 2a Avaliação \\
\hline $0,80-0,89$ & 73,3 & 64,0 & 51,0 \\
$0,90-1,00$ & 36,0 & $49, .0$ \\
\hline
\end{tabular}

* Cálculo baseado no número de ocorrências no intervalo comparado ao total de 45 combinações possíveis entre os 10 avaliadores em cada avaliação da severidade da doença.

Os níveis de acurácia, precisão e reprodutibilidade das mensurações da severidade da antracnose em vagens de feijão-fava não melhoraram significativamente com o uso da escala diagramática para quantificação da antracnose em vagens de feijãocomum. Esses resultados diferem dos constatados com a utilização de outras escalas diagramáticas, pois todos demonstraram a importância dessa ferramenta em estudos de quantificação de doenças (Kranz, 1988; Campbell e Madden, 1990; Madden et al., 2007; Bock et al., 2010; Duarte et al., 2014; Lopes et al., 2014; Michereff e Correia, 2016). Diferenças na morfologia das vagens do feijoeiro em relação às vagens do feijãocomum, bem como os distintos padrões de distribuição das lesões, podem explicar, pelo menos em parte, a ineficácia da utilização da escala desenvolvida por 
Castaño-Zapata (1989) para avaliação da antracnose em vagens de feijão-fava. Dessa forma a utilização da escala avaliada em estudos epidemiológicos da antracnose do feijão-fava poderá não reduzir a subjetividade das estimativas e propiciar informações pouco precisas sobre a intensidade da doença. Diante disso, torna-se essencial o desenvolvimento e a validação de uma escala diagramática específica para quantificação da antracnose em vagens de feijão-fava.

\section{AGRADECIMENTOS}

Os autores expressam seus agradecimentos aos avaliadores que participaram das etapas de validação da escala diagramática.

\section{REFERÊNCIAS BIBLIOGRÁFICAS}

Barbosa, M.A.G.; Michereff, S.J.; Mora-Aguilera, G. Elaboração e validação de escala diagramática para avaliação da severidade da ferrugem branca do crisântemo. Summa Phytopathologica, 2006, 32, 1, 5762.

Bock, C.H.; Poole, G.; Parker, P.E.; Gottwald, T.R. Plant disease severity estimated visually, by digital photography and image analysis, and by hyperspectral imaging. Critical Reviews in Plant Sciences, 2010, 29, 59-107.

Buffara, C.R.S.; Angelotti, F.; Vieira, R.A.; Bogo, A.; Tessmann, D.J.; Bem, B.P. Elaboration and validation of a diagrammatic scale to assess downy mildew severity in grapevine. Ciência Rural, 2014, 44, 8, 1384-1391.

Campbell, C.L.; Madden, L.V. Introduction to plant disease epidemiology. New York: John Wiley, 1990. 532 p.

Carmo,M.D.S.; Carvalho, E.M.S.; Gomes, R.L.F.;Lopes, A.C.A.; Cavalcante, G.R.S. Avaliação de acessos de feijão-fava, para resistência a Colletotrichum truncatum, em condições de folhas destacadas e campo. Summa Phytopathologica, 2015, 41, 4, 292-297.

Carvalho, E.M.S. Antracnose em feijão-fava: caracterização do agente causal e reação de genótipos a Colletotrichum truncatum. 2009. 69f. Tese (Doutorado em Agronomia - Produção Vegetal) - Universidade Estadual Paulista "Júlio de Mesquita Filho", Jaboticabal.
Castaño-Zapata, J. Estandarización de la estimación de daños causados por hongos, bacterias y nematodos en frijol (Phaseolus vulgaris L.). Fitopatologia Colombiana, 1989, 13, 1, 9-19.

Cavalcante, G.R.S.; Carvalho, E.M.S.; Gomes, R.L.F.; Santos, A.R.B.; Santos, C.M.P.M. Reação de subamostras de feijão-fava à antracnose. Summa Phytopathologica, 2012, 38, 4, 329-333.

Celoto, M.I.B.; Papa, M.F.S. Elaboração e validação de escala diagramática para quantificação da mancha alvo em folhas de acerola. Tropical Plant Pathology, 2010, $35,4,258-262$.

Duarte, H.S.S.; Capucho, A.S.; Lopes, U.P.; Zambo$\lim$, L. Elaboração e validação de escala diagramática. In: Zambolim, L.; Jesus Júnior, W.C.; Rodrigues, F.A. (Eds.). $O$ essencial da Fitopatologia: epidemiologia de doenças de plantas. Viçosa: Universidade Federal de Viçosa, 2014. p. 81-97.

Guimarães, W.N.; Martins, L.S.; Silva, E.F.; Ferraz, G.M.G.; Oliveira, F.J. Caracterização morfológica e molecular de acessos de feijão-fava (Phaseolus Iunatus L.). Revista Brasileira de Engenharia. Agrícola e Ambiental, 2007, 11, 1, 37-45.

IBGE. SIDRA - sistema IBGE de recuperação automática. Rio de Janeiro: Fundação Instituto Brasileiro de Geografia e Estatística, 2016. Disponível em: <http:/l www.sidra.ibge.gov.br>. Acesso em: 25 out. 2016.

Kranz, J. Measuring plant disease. In: Kranz, J.; Rotem, J. (Ed.). Experimental techniques in plant disease epidemiology. Heidelberg: Springer-Verlag, 1988. p. 35-50.

Lamari, L. ASSESS 2.0: Image analysis software for plant disease quantification. St. Paul: APS Press, 2008. 125 p.

Leite, R. M.V.B.C.; Amorim, L. Elaboração e validação de escala diagramática para mancha de Alternaria em girassol. Summa Phytopathologica, 2002, 28, 1, 14-19.

Lima, G.S.A.; Assunção, I.P.; Martins, R.B.; Santos, H.V.; Michereff, S.J. Development and validation of a standard area diagram set for assessment of Alternaria spot on the cladodes of the prickly pear cactus. Journal of Plant Pathology, 2011, 93, 3, 691-695. 
Lopes, U.P.; Duarte, H.S.S.; Capucho, A.S.; Zambolim, L. Quantificação de doenças de plantas. In: Zambolim, L.; Jesus Júnior, W.C.; Rodrigues, F.A. (Eds.). 0 essencial da Fitopatologia: epidemiologia de doenças de plantas. Viçosa: Universidade Federal de Viçosa, 2014. p. 51-77.

Madden, L.V.; Hughes, G.; van den Bosch, F. The study of plant disease epidemics. St. Paul: APS Press, 2007. $421 \mathrm{p}$.

Michereff, S.J.; Correia, K.C. Quantificação de fitobacteriores. In: Gama, M.A.S.; Nicoli, A.; Guimarães, L.M.P.; Lopes, U.P.; Michereff, S.J. (Eds.). Estado da arte em fitobacterioses tropicais. Recife: EDUFRPE, 2016. p. 83-106.

Nutter Jr., F.W.; Schultz, P.M. Improving the accuracy and precision of disease assessments: selection of methods and use of computer-aided training programs. Canadian Journal of Plant Pathology, 1995, 17, 1, 174184.

Paula Júnior, T.J.; Silva, M.B.; Vieira, R.F. Doenças causadas por fungos em hortaliças leguminosas. Informe Agropecuário, 1995, 17, 182, 63-71.

Silva, K.J.D.; Lopes, A.C.A.; Gomes, R.L.F.; Sousa, F.M.; Rocha, M.M. Recursos genéticos. In: Lopes, A.C.A.; Gomes, R.L.F.; Araújo, A.S.F. (Eds.). A cultura do feijão-fava no Meio-Norte do Brasil. Teresina: Editora da UFPI, 2010. $272 \mathrm{p}$.

Sposito, M.B.; Amorim, L.; Belasque Junior, J.; Bassanezi, R.B.; Aquino, R. Elaboração e validação de escala diagramática para avaliação da severidade da mancha preta em frutos cítricos. Fitopatologia Brasileira, 2004, $29,1,81-85$. 